\title{
Berg, Sabrina
}

\section{Pädagogische Praxis und Reproduktion sozialer Ungleichheit - zur} \section{Berücksichtigung sozialer Herkunft im Wirtschaftsunterricht}

Seifried, Jürgen [Hrsg.]; Faßhauer, Uwe [Hrsg.]; Seeber, Susan [Hrsg.]: Jahrbuch der berufs- und wirtschaftspädagogischen Forschung 2014. Opladen [u.a.] : Verlag Barbara Budrich 2014, S. 161-173. (Schriftenreihe der Sektion Berufs- und Wirtschaftspädagogik der Deutschen Gesellschaft für Erziehungswissenschaft (DGfE); 217)

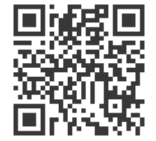

Quellenangabe/ Reference:

Berg, Sabrina: Pädagogische Praxis und Reproduktion sozialer Ungleichheit - zur Berücksichtigung sozialer Herkunft im Wirtschaftsunterricht - In: Seifried, Jürgen [Hrsg.]; Faßhauer, Uwe [Hrsg.]; Seeber, Susan [Hrsg.]: Jahrbuch der berufs- und wirtschaftspädagogischen Forschung 2014. Opladen [u.a.] : Verlag Barbara Budrich 2014, S. 161-173 - URN: urn:nbn:de:0111-pedocs-97331 - DOI: 10.25656/01:9733

https://nbn-resolving.org/urn:nbn:de:0111-pedocs-97331

https://doi.org/10.25656/01:9733

in Kooperation mit / in cooperation with:

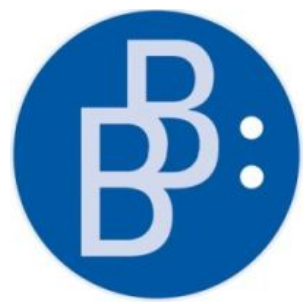

https://www.budrich.de

\section{Nutzungsbedingungen}

Dieses Dokument steht unter folgender Creative Commons-Lizenz:

http://creativecommons.org/licenses/by-nc-nd/3.0/de/deed - Sie dürfen das Werk bzw. den Inhalt unter folgenden Bedingungen vervielfältigen, verbreiten und öffentlich zugänglich machen: Sie müssen den Namen des Autors/Rechteinhabers in der von ihm festgelegten Weise nennen. Dieses Werk bzw. dieser Inhalt darf nicht für kommerzielle Zwecke verwendet werden und es darf nicht bearbeitet, abgewandelt oder in anderer Weise verändert werden.

Mit der Verwendung dieses Dokuments erkennen Sie die Nutzungsbedingungen an.

\section{Terms of use}

This document is published under following Creative Commons-License: http://creativecommons.org/licenses/by-nc-nd/3.0/de/deed.en - You may copy, distribute and transmit, adapt or exhibit the work in the public as long as you attribute the work in the manner specified by the author or licensor. You are not allowed to make commercial use of the work or its contents. You are not allowed to alter, transform, or change this work in any other way.

By using this particular document, you accept the above-stated conditions of use.

\section{Kontakt / Contact:}

\section{peDOCS}

DIPF | Leibniz-Institut für Bildungsforschung und Bildungsinformation

Informationszentrum (IZ) Bildung

E-Mail: pedocs@dipf.de

Internet: www.pedocs.de

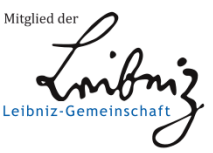




\section{Jahrbuch der berufs- und wirtschaftspädagogischen Forschung 2014}

Jürgen Seifried, Uwe Faßhauer Susan Seeber (Hrsg.)

DGE Deutsche Gesellschaft 
Schriftenreihe der Sektion

Berufs- und Wirtschaftspädagogik der Deutschen Gesellschaft für Erziehungswissenschaft (DGfE) 
Jürgen Seifried

Uwe Faßhauer

Susan Seeber (Hrsg.)

\section{Jahrbuch der berufs- und wirtschaftspädagogischen Forschung 2014}

Verlag Barbara Budrich

Opladen • Berlin • Toronto 2014 
Bibliografische Information der Deutschen Nationalbibliothek

Die Deutsche Nationalbibliothek verzeichnet diese Publikation in der Deutschen Nationalbibliografie; detaillierte bibliografische Daten sind im Internet über http://dnb.d-nb.de abrufbar.

(C) Dieses Werk ist im Verlag Barbara Budrich erschienen und steht unter folgender Creative Commons Lizenz: http://creativecommons.org/licenses/by-nc-nd/3.0/de/ Verbreitung, Speicherung und Vervielfältigung erlaubt, kommerzielle Nutzung und Veränderung nur mit Genehmigung des Verlags Barbara Budrich.

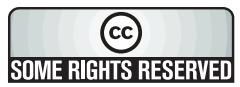

Dieses Buch steht im OpenAccess Bereich der Verlagsseite zum kostenlosen Download bereit (http://dx.doi.org/10.3224/84740164)

Eine kostenpflichtige Druckversion (Printing on Demand) kann über den Verlag bezogen werden. Die Seitenzahlen in der Druck- und Onlineversion sind identisch.

$$
\begin{array}{ll}
\text { ISBN } & 978-3-8474-0164-3 \text { (Paperback) } \\
\text { eISBN } & \mathbf{9 7 8 - 3 - 8 4 7 4 - 0 4 4 1 - 5 ~ ( e B o o k ) ~} \\
\text { DOI } & 10.3224 / 84740164
\end{array}
$$

Umschlaggestaltung: bettina lehfeldt graphic design, Kleinmachnow

Typografisches Lektorat: Judith Henning, Hamburg

Verlag Barbara Budrich, http://www.budrich-verlag.de 


\section{Inhaltsverzeichnis}

Vorwort 9

\section{Teil I: Perspektiven der historischen Berufsbildungsforschung}

Frank-Lothar Kroll

Möglichkeiten und Notwendigkeiten historiographischen Arbeitens in der Berufs- und Wirtschaftspädagogik........................................................ 1

Volker Bank, Annekathrin Lehmann

Theodor Franke. Sächsischer Pionier wirtschaftspädagogischen

Denkens in Deutschland

Marcel Schweder

Lehrerarbeit im Strafvollzug - Ein Diskurs aus historischer Sicht

\section{Teil II: Kompetenzmodellierung, -messung und -förderung}

Eveline Wittmann, Ulrike Weyland, Annette Nauerth, Ottmar Döring, Simone Rechenbach, Julia Simon, Iberé Worofka Kompetenzerfassung in der Pflege älterer Menschen - Theoretische und domänenspezifische Anforderungen der Aufgabenmodellierung 53

Simon Heinen, Martin Frenz, Christopher M. Schlick

Bildung für nachhaltige Entwicklung in der Gebäudeenergieberatung - Entwicklung eines Kompetenzmodells für die Förderung reflexiver Handlungsfähigkeit

Diana Stuckatz, Cornelia Wagner

Qualifizierungsangebote in der Pflegehilfe für Personen mit geringen Grundbildungskenntnissen - Empirische Studien zur Entwicklung von Lehr-Lern-Umgebungen und Arbeitsmaterialien. 81 


\section{Teil III: Gestaltung und Analyse von Lehr-Lern-Prozessen}

Eva Höpfer, Andrea Reichmuth, Doreen Holtsch, Franz Eberle

Wer sieht was? - Zum Umgang mit unterschiedlichen Sichtweisen auf

Unterricht am Beispiel des kaufmännischen Berufsschulunterrichts 95

Mandy Hommel

Sozial geteilte Reflexion - eine explorative Studie im

Mathematikunterricht. 109

Gerhard Minnameier, Rico Hermkes

„Kognitive Aktivierung“ und „konstruktive Unterstützung“ als Lehr-

Lern-Prozess-Größen - Eine Konzeption im rechnungswesen-

didaktischen Kontext

\section{Teil IV: Lehrerbildung und pädagogische Professionalität}

Nicole Kimmelmann, Johannes Lang

Lehramtsstudierende mit Migrationshintergrund und ihre

Schwierigkeiten an der Universität

Robert W. Jahn

Stützlehrer als neuer pädagogischer Profi in der Beruflichen Bildung?! .... 147

Sabrina Berg

Pädagogische Praxis und Reproduktion sozialer Ungleichheit - zur

Berücksichtigung sozialer Herkunft im Wirtschaftsunterricht..... 161

\section{Teil V: Perspektiven der Berufsbildungsforschung}

\section{Miriam Voigt}

Neo-institutionalistische und mikropolitische Prozesse in

Schulentwicklungsprojekten

Lara Forsblom, Lucio Negrini, Jean-Luc Gurtner \& Stephan Schumann

Lehrvertragsauflösungen und die Rolle der betrieblichen Auswahl von

Auszubildenden 
Marius R. Busemeyer

Organisierte Interessen, Parteipolitik und institutioneller Wandel im deutschen Berufsbildungssystem

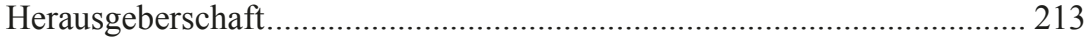

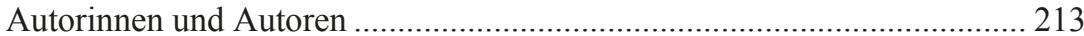




\section{Pädagogische Praxis und Reproduktion sozialer Ungleichheit - zur Berücksichtigung sozialer Herkunft im Wirtschaftsunterricht}

Sabrina Berg

\section{Einleitung}

Im vorliegenden Beitrag wird der Frage nachgegangen, auf welche Weise in der Praxis des Wirtschaftsunterrichts die soziale Herkunft von Schüler_innen durch unterrichtliche Konstruktion der Lehrkräfte relevant wird. Dabei wird der Frage nachgespürt, ob Lehrkräfte soziale Herkunft überhaupt als relevanten Faktor für unterrichtliches Handeln wahrnehmen. Annahme ist, dass die Konstruktion von Unterricht und die damit einhergehenden handlungsleitenden Denk-, Wert- und Urteilsmuster auch mit der sozialen Herkunft der Lehrkräfte in Verbindung stehen. Erkenntnisinteresse ist, ob sich hieraus auf Indizien zur Reproduktion sozialer Ungleichheit in und durch pädagogische Praxis im Wirtschaftsunterricht schließen lässt. Im Beitrag wird die Perspektive der Lehrkräfte fokussiert. Diese sind herausgefordert, über didaktische Konstruktion wirtschaftliche Inhalte lebensweltlich konkret aufzubereiten. Dabei sind sowohl sie selbst als auch die Schüler_innen durch eine herkunftsspezifische Handlungslogik geleitet. Das Aufeinandertreffen der subjektiven Deutungsmuster im Wirtschaftsunterricht lässt sich damit als Problem der kulturellen Passung zwischen Herkunfts- und Schulmilieu fassen (vgl. Kramer/Helsper 2011, S. 105). Aus rein fachdidaktischer Perspektive erschließen sich die Mechanismen im Lehr-Lernprozess, die diese Passungsproblematik verursachen, nicht unmittelbar. Darum wird der Blick auf den bildungssoziologischen Diskurs gelenkt und diese Mechanismen mit der Kulturtheorie von Bourdieu theoretisch hinterlegt. Hierüber lassen sich Faktoren der Reproduktion sozialer Ungleichheit sowie die Passungsproblematik in schulischen Kontexten beschreiben. Im Beitrag wird die Linie lehrkraftseitig vorhandener subjektiver Theorien in pointierter Weise auf das Spannungsfeld zwischen meritokratischem Anspruch und inhaltlicher Umsetzung bezogen. Dies wird vor dem Hintergrund der Reproduktion sozialer Ungleichheit im 
Fach diskutiert. ${ }^{1}$ Dabei wird die Frage, welche Rolle die soziale Herkunft der Schüler_innen als unterrichtswirksames Element im Wirtschaftsunterricht spielt, auf theoretischer Ebene diskutiert. Der empirische Teil dieses Beitrags zielt auf die Fragestellung ab, ob Lehrkräfte die soziale Herkunft der Schüler_innen als relevanten Faktor im Wirtschaftsunterricht wahrnehmen. Die Argumentation verläuft in vier Schritten: Zunächst wird in einem Problemaufriss das Erkenntnisinteresse dargestellt, danach in der theoretischen Rahmung a) auf die Rolle der lernenden Subjekte im Wirtschaftsunterricht, b) auf die Rolle der lehrenden Subjekte und c) auf die Reproduktion sozialer Ungleichheit im Lehr-Lernprozess des Wirtschaftsunterrichts eingegangen. Anschließend werden Ausschnitte episodischer Interviews mit Lehrkräften berufsbildender Schulen auf Grundlage der theoretischen Rahmung interpretiert und diskutiert.

\section{Problemaufriss}

Von der Schule als „Chancenverteilungsstelle“ (vgl. Bauer 2011) wird erwartet, dass sie gleiche Ausgangslagen für Schulerfolg von Schüler_innen unabhängig von der - in diesem Fall - sozialen Herkunft ermöglicht. Ausgangspunkt der Betrachtung ist, dass Schule nur in Teilen soziale Durchlässigkeit ermöglicht und somit eine „meritokratische Illusion“ (vgl. Geißler 2012) darstellt, wobei über das Bildungssystem soziale Ungleichheit reproduziert und produziert wird. Als Faktoren, die hierbei eine Rolle spielen, gelten: das Schulsystem, mangelnde Orientierung von Bildungsprozessen an bildungsfernen Schichten durch die „Mittelschichtsorientierung“ der Schule und schließlich die Lehrkräfte selbst (Ditton 2007, S. 265). Diese diskriminieren zwar nicht bewusst, ihre impliziten Persönlichkeits- und Begabungstheorien sind aber darauf zu prüfen, ob sie Kinder aus unteren sozialen Klassen benachteiligen (ebd.). Die Erwartungen, die Schule an Schüler_innen stellt, entsprechen bezüglich bestimmter Verhaltensweisen, Umgangsformen oder des nachzuweisenden Könnens eher dem Lebenskontext und dem Habitus oberer sozialer Gruppen (vgl. Ditton 2009). „Kinder der oberen Statusgruppen sind sozusagen kompatibler mit dem, was in der Schule erwartet und honoriert wird. Ebenso spiegelt sich in den Wahrnehmungen und Urteilen von Lehrkräften über Schüler ein gewisser sozialer ,bias' wider, etwa in der Einschätzung der Begabungen und des intellektuellen Potentials der Schüler" (a.a.O.:

1 Die Veröffentlichung basiert auf dem Dissertationsprojekt der Autorin. In Berg (2014) wird die kulturtheoretische Hinterlegung der Passungsproblematik zwischen Lehrkräften und Schüler_innen (hier: Kapitel 3) in einen breiteren Zusammenhang mit sozialisationstheoretischer Rahmung auf die Anknüpfungsfähigkeit von Lerngegenständen an die Lebenswelt der lernenden Subjekte im Fach gestellt. 
S. 239). Damit ergibt sich der Diskurs um soziale Ungleichheiten im Bildungssystem als Ausgangspunkt für den vorliegenden Beitrag. Dieser wird mit Bezug auf subjektive Theorien von Lehrkräften im Wirtschaftsunterricht diskutiert.

Für den Bereich der ökonomischen Bildung stellen Tests zur Messung ökonomischen Wissens oder auch die kognitions- und sozialpsychologische Forschung Unterschiede im ökonomischen Wissen und Verständnis verschiedener Gruppen fest (vgl. Birke/Seeber 2011, S. 57). Heterogenität in Bezug auf das ökonomische Verständnis wurde bezüglich Lernergruppen mit unterschiedlichem sozioökonomischem Status untersucht und nachgewiesen (vgl. Seeber/Remmele 2009, S. 31 mit Bezug auf Roland-Lévy 2002). Im Zugang zu ökonomischen Themen kann damit von herkunftsspezifischem Vorwissen und Vorprägungen der Schüler_innen ausgegangen werden.

Als ein handlungsleitendes Konzept für den Wirtschaftsunterricht gilt die Lebenssituationsorientierung (vgl. Retzmann et al. 2010, S. 122). Hier sollen Lehrkräfte die lebensweltlichen Dimensionen des Wirtschaftens für praktisch relevante Zusammenhänge der Schülerinnen und Schüler fachdidaktisch aufbereiten (ebd.). In diesen Lehr-Lernsituationen werden die Subjekte auf die Bewältigung sozio-ökonomischer Lebenssituationen vorbereitet (vgl. Euler/Hahn 2007, S. 75ff.). Denkt man im Zusammenhang mit der Lebenssituationsorientierung an die soziale Herkunft der Lernenden wie auch der Lehrenden, hat man es mit einem doppelten Konstruktionsprozess zu tun (vgl. Berg 2014, S. 2): Einerseits bringt das pädagogische Personal selbst subjektive Vorstellungen in die Gestaltung des Lehr-Lernprozesses ein (vgl. Dubs 2001). Andererseits bringen auch die Lernenden ihre subjektiven Alltagsvorstellungen ein (vgl. Aprea 2011, S. 100). Gerade ökonomische Unterrichtsgegenstände sind schon sozialisationsbedingt lebensweltlich konkret - Lernende verfügen über informelle Lernerfahrungen. Im Wirtschaftsunterricht treffen diese beiden im sozialen Herkunftsmilieu entstandenen Vorstellungen, aufeinander. Sind Lerngegenstand und subjektive Vortellungen sowie Wissen darüber seitens der lernenden Subjekte herkunftsspezifisch heterogen und ist dies gleichzeitig als blinder Fleck in der Fachdidaktik anzusehen, so stellt sich auf Ebene des Wirtschaftsunterrichts die Frage nach den handlungsleitenden Mechanismen der Lehrenden und der Reproduktion sozialer Ungleichheiten über unterrichtliches Handeln. 


\section{Theoretische Rahmung}

\subsection{Das lernende Subjekt}

Alltagsvorstellungen der Lernenden werden unter Bezeichnungen wie subjektive Theorien (vgl. z.B. Groeben et. al. 1988) eine zentrale Rolle im lernpsychologischen und didaktischen Diskurs zugesprochen (vgl. Aprea 2011, 100). Vorstellungen, die sich Lernende von der Welt machen, bringen sie als „kognitives Gepäck“ in institutionalisierte Lernprozesse ein. Alltagsvorstellungen können dabei sowohl lernförderlich wirken als auch zum Lernhindernis werden oder zu Lernwiderständen führen, wenn sie nicht anschlussfähig sind (ebd.). Mechanismen und Prozesse der emotionalen Befindlichkeit wirken sowohl für Schüler_innen als auch für Lehrkräfte. Wird ausschließlich auf die kognitive Dimension von Unterricht fokussiert, führt dies zwangsläufig zu Beeinträchtigungen im Lehr-Lernprozess in Form von Dysfunktionalitäten und Konflikten zwischen den Beteiligten (vgl. Seifried/Sembill 2005, S. $658)$.

\subsection{Das lehrende Subjekt}

Für die Lehrenden beschreibt Dubs (2001) zur Handelslehrerausbildung das „produktive Spannungsfeld“ zwischen Wissenschaftsorientierung und Praxisbezug. Einerseits könnte argumentiert werden, das Schwergewicht der Lehrerbildung sei auf ein wissenschaftlich gestaltetes pädagogisches Inhaltsund Handlungswissen zu legen, das die Grundlage für Entscheidungen im Schulalltag gebe, so dass eine kurze praktische Ausbildung genüge, um aus der Erfahrung die notwendigen Handlungskompetenzen relativ leicht zu erwerben. Andererseits könnte argumentiert werden, dass das Entscheidende im Lehrerberuf pädagogische Handlungskompetenzen, also vor allem Fertigkeiten in der Unterrichtsgestaltung seien. Für diese reichten als Grundlage „naive Theorien“, also Aussagesysteme, die ausschließlich auf der praktischen Erfahrung aufbauen (vgl. Dubs 2001, S. 4). Auch Seifried (2009) kommt zu dem Schluss, dass subjektive Theorien den wissenschaftlichen Theorien nicht zwingend unterlegen sind, gleichwohl unterscheiden sie sich in ihrer Leistungsfähigkeit, wobei keine prinzipielle Überlegenheit der wissenschaftlichen Theorien anzunehmen ist. Je nach Zielsetzung können auch subjektive Alltagstheorien ausreichend sein (a.a.O.: S. 84f.). Wenn aber subjektive Theorien über Sozialisationseinflüsse vor dem Hintergrund von kulturellen Einflüssen entstehen (a.a.O.: S. 85) stellt sich für den Bereich der sozialen Herkunft der Subjekte als blinden Fleck der Fachdidaktik die Frage danach, wie das unterrichtliche Handeln nach subjektiven Theorien gestaltet 
wird. Sind diese durch die eigene Sozialisation klassenspezifisch geprägt, könnte dies zur Reproduktion sozialer Ungleichheit auf Ebene der Unterrichtspraxis führen.

\subsection{Die Reproduktion sozialer Ungleichheit im Wirtschaftsunterricht}

Um die prinzipielle Hinterlegung in Bezug auf Reproduktion sozialer Ungleichheit im Wirtschaftsunterricht genauer beleuchten zu können, wird die Argumentationslinie mit Bourdieu bildungssoziologisch erweitert. Darüber lässt sich eine Öffnung hinsichtlich der strukturellen Handlungslogiken der Subjekte vor dem Hintergrund der jeweiligen Klassenzugehörigkeit und der kulturellen Passung zueinander - auch für den Lehr-Lernprozess - genauer fassen. Mit Bourdieu erfolgen die Betrachtungen aus einer praxeologischen Perspektive, die nach den Handlungslogiken der Subjekte fragt und die „Logik der Praxis" als zentralen Handlungsmoment ansieht. Als Ausgangspunkt lassen sich in diesem Zusammenhang der Habitus der Lernenden und Lehrenden und Bourdieus Ausführungen zum sozialen Raum bestimmen.

\subsubsection{Zum sozialen Raum}

Der Begriff des Raums wird bei Bourdieu als passive Leinwand, in die sich soziale Strukturen einschreiben, angesehen. Dieser gilt gerade im Kontext mit sozialer Ungleichheit als Faktor für die Verfestigung sozialer Ungleichheitsstrukturen (vgl. Schroer 2008, S. 155f.). Der soziale Raum stellt eine relationistische Konzeption der sozialen Welt dar, welchem „bestimmte Unterscheidungs- und Verteilungsprinzipien zugrunde liegen“ (Bourdieu 1995, S. 9). Unterschiede in diesem Raum konstituieren sich über das Vorhandensein von Kapital, welches des Akteuren und Gruppen nicht in gleicher Weise zur Verfügung steht (vgl. Kramer 2011, S. 42). Die Akteure, Gruppen, Institutionen im sozialen Raum weisen umso mehr gemeinsame Merkmale auf, je näher sie zueinander in diesem Raum sind; umso weniger gemeinsame Merkmale, je ferner sie sich sind (vgl. Bourdieu 1992, S. 139). Die sich im sozialen Raum nahestehenden Personen stehen sich zwar willentlich oder gezwungenermaßen tendenziell auch geographisch nahe, gleichwohl können sich auch sozial fern stehende Menschen zumindest kurzfristig und mit Unterbrechungen im physischen Raum treffen und interagieren (ebd.).

Wenn davon auszugehen ist, dass sich sozial nahe stehende Personen auch vermehrt miteinander interagieren stellt sich für die Betrachtung des sozialen Raums Schule hier die Frage nach dem Lehrer-Schüler-Verhältnis hinsichtlich der jeweiligen sozialen Herkunft (vgl. Berg 2014, S. 6f.). Lehrkräfte sind hauptsächlich einem bildungsbürgerlichen Milieu zuzuordnen, wie Bourdieu schon für die Volksschul- und Gymnasiallehrer Frankreichs 
zeigen konnte (vgl. Bourdieu 2012, S. 212f.). Auch für Deutschland sind entsprechende Untersuchungen erfolgt (vgl. z.B. Kühne 2006). „Lehrkräfte sind demzufolge per Profession dazu verpflichtet in geographischer Nähe im sozialen Raum mit Personen zu interagieren, mit denen sie jenseits ihrer pädagogischen Profession selten oder eher zufällig in Kontakt treten würden“" (Berg 2014, S. 7). Dies ist Ansatzpunkt für die Frage nach der kulturellen Passung im Wirtschaftsunterricht. Die Ausführungen zum sozialen Raum alleine lösen die Frage nach den Handlungslogiken der lehrenden Subjekte aber nicht auf. Darum wird im Folgenden auf das Konzept des Habitus Bezug genommen.

\subsubsection{Zum Habitus}

In der sozialen Welt existieren objektive Strukturen, die unabhängig vom Bewusstsein und Willen der Handelnden sind, jedoch deren Praktiken und Vorstellungen leiten. So konstituiert sich der Habitus über eine soziale Genese sowohl der Wahrnehmungs-, Denk-, und Handlungsschemata als auch der sozialen Strukturen (vgl. Bourdieu 1992, S. 135). Davon ausgehend werden auch im Wirtschaftsunterricht sowohl die Praktiken und Vorstellungen der Subjekte als auch die sozialen Strukturen produziert und reproduziert. Verschiedenen Klassen innerhalb der Gesellschaft wohnen unterschiedliche Wahrnehmungs-, Denk- und Handlungsformen inne, wodurch sich Klassen, auch in ihrer Unterschiedlichkeit, stets reproduzieren (vgl. Joas/Knöbl 2011, S. 534). Die Vermittlung zwischen den wahrnehmbaren Verhaltensformen und dem System vollzieht sich über den Habitus. Dieser wird verstanden als System der organischen und mentalen Dispositionen und Denk-, Wahrnehmungs-, und Handlungsschemata. Er bedingt damit die Erzeugung der Wahrnehmungen und Handlungen die zunächst spontan erscheinen, jedoch beobachtbaren Regelmäßigkeiten entsprechen (vgl. Bourdieu 1974, S. 39-41). Aus dieser Perspektive wird eine ständige Reproduktion sozialer Ungleichheit durch das Bildungswesen, das immer wieder klassenspezifische Verhaltensformen verstärkt, beschrieben (vgl. Joas/Knöbl 2011, S. 548).

Mit Bourdieu wird davon ausgegangen, dass die den Lehr-Lernprozess determinierenden sozialen Strukturen, und Denk- Wert- und Urteilsmuster der Lehrkräfte auf implizit durch den Habitus vorgegebene Vorstellungen von den Schüler_innen zurückzuführen sind (vgl. Berg 2014, S. 6). „Die sozialen Praktiken die hieraus seitens der Lehrkräfte im Unterricht folgen, werden zwar bewusst vorgenommen, ihre Strukturierung verläuft jedoch

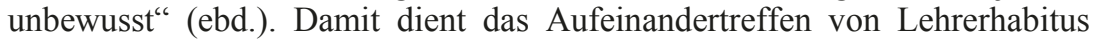
und Schülerhabitus im sozialen Raum als Interpretationschema für die ausgewählten empirischen Ergebnisse. 


\section{Empirie}

\subsection{Methodisches Vorgehen}

Zielgruppe der Befragung sind Lehrkräfte der berufsbildenden Schulen die das Fach Wirtschaft in unterschiedlichen Bildungsgängen unterrichten und dementsprechend mit Schüler_innen verschiedenster sozialer Herkunft in Kontakt treten. Bei der Auswahl der Befragten wurde auf die folgenden Punkte Wert gelegt: Die Lehrkräfte sind an einer berufsbildenden Schule mit dem Schwerpunkt Wirtschaft tätig, unterrichten das Fach Wirtschaft in unterschiedlichen Bildungsgängen und es besteht Heterogenität hinsichtlich der Beschäftigungszeit. Zudem wurde der Faktor „Stadt-Land“ berücksichtigt. Es wurden sechs episodische Interviews nach Flick (2008) geführt. ${ }^{2}$ Der Leitfaden umfasst Fragen zu den Bereichen (I) eigene Berufsbiographie, (II) Wirtschaftsunterricht und (III) soziale Herkunft. Dabei wurden sowohl Fragen die auf semantisches Wissen als auch solche die auf episodisches Wissen abzielen, gestellt. Über diese Verbindung können soziale Repräsentationen als eine Mischform zwischen individuellem und sozialem Denken und Wissen (vgl. Flick 2008, S. 37) auf die Fragestellung des Beitrags bezogen werden.

Zur Analyse der Daten wurde das Material nach Flick (2008) in Anlehnung Strauss (1991) thematisch kodiert. Über das in Einzelfallanalyse entwickelte Kategoriensystem wurden fallübergreifende Kategorien verglichen und hinsichtlich der Gemeinsamkeiten und Unterschiede ausgewertet. So bezieht sich die Darstellung der Ergebnisse und Interviewausschnitte in Kapitel 4.2 auf das anschließende Zusammenfassen von Gemeinsamkeiten innerhalb der Fälle (vgl. Flick 2012, S. 405). Dabei werden ausgewählte Interviewpassagen zum Gegenstandsbereich „Relevanzsetzung der Lehrkräfte zur sozialen Herkunft der Schüler_innen“" herausgestellt und vor dem Hintergrund der theoretischen Rahmung diskutiert.

\subsection{Ausgewählte Ergebnisse}

Im Folgenden werden ausgewählte Ergebnisse des fallübergreifenden Vergleichs in den Kategorien „Relevanz sozialer Herkunft“, „Gleichbehandlung“ und „Einbezug sozialer Herkunft“ dargestellt.

2 Die episodischen Interviews wurden im Rahmen des Dissertationsprojekts der Autorin geführt. Teile des Datenmaterials werden in Berg (2014) mit dem Fokus auf das lernende Subjekt im Fach diskutiert. 


\subsubsection{Relevanz sozialer Herkunft}

Zunächst zur Frage, ob die soziale Herkunft die Gestaltung des eigenen Wirtschaftsunterrichts beeinflusst. In einem fallübergreifenden Vergleich lässt sich als Gemeinsamkeit herausstellen, dass die befragten Lehrkräfte der Meinung sind, dass die soziale Herkunft der Schüler_innen für ihre eigene Gestaltung des Wirtschaftsunterrichts keine Relevanz hat bzw. haben sollte. Exemplarisch für die Kategorie gilt die folgende Aussage von Lehrkraft C:

„Das darf keinen Stellenwert haben! [...] Es darf keinen haben, dass wir darauf sozusagen gucken kann ich mir nicht vorstellen, dass Kolleginnen und Kollegen aber vielleicht bin ich jetzt auch ideal ein bisschen idealistisch in der Hinsicht. Aber natürlich ist, spielt das eine Rolle, keine Frage aber es, wir sind dafür da, dass eben das eigentlich keine Rolle spielt und dass wir so versuchen die Position ja so die Schülerinnen und Schüler ja so in eine Position zu bringen dass das keine Rolle spielt aus, welche Herkunft man hat. Das wäre jedenfalls mein Ansatz. Das darf keine, es darf keine Rolle spielen und wir müssen uns bemühen jeden Einzelnen in eine Lage zu versetzen beruflich erfolgreich später tätig zu werden oder auch anders tätig zu werden."

Die befragten Lehrkräfte sind der Meinung, dass die soziale Herkunft der Schüler_innen für ihre Gestaltung des Wirtschaftsunterrichts keine Rolle spielen sollte. Gleichwohl sind sie implizit der Auffassung, dass die soziale Herkunft relevant sein könnte (siehe die Hervorhebung im Zitat).

Dabei werden diese Äußerungen zunächst nicht mit einem situativen Kontext verknüpft. Deutlich wird im fallübergreifenden Vergleich, dass dieser Faktor offenbar negativ belegt ist, denn Einigkeit besteht darüber, dass das eigentlich lieber nicht so sein sollte. Exemplarisch dafür ist eine Aussage bei Lehrkraft $\mathrm{C}$, ,vielleicht bin ich jetzt auch ideal ein bisschen idealistisch“ und die Einigkeit darüber, dass soziale Herkunft eigentlich besser keine Relevanz erfahren sollte, herausgestellt werden kann (vgl. Berg 2014, S. 11).

\subsubsection{Gleichbehandlung}

Als weitere Gemeinsamkeit lässt sich herausstellen, dass die Äußerungen da$\mathrm{zu}$, dass soziale Herkunft nicht relevant für den eigenen Wirtschaftsunterricht ist, mit dem Anspruch einhergeht, alle Schüler_innen gleich zu behandeln. Exemplarisch für die Kategorie gilt folgende Äußerung von Lehrkraft E:,,Nein, also eigentlich nicht, wir behandeln alle gleich. " Darüber kommt zum einen der lehrkraftseitige Anspruch an meritokratische Prinzipien (vgl. Kap. 2) auch im Wirtschaftsunterricht zum Tragen. Zum anderen stellt dies wiederum die negative Belegung der sozialen Herkunft als relevanten Faktor im Wirtschaftsunterricht heraus, denn mit der Relevanz von sozialer Herkunft 
der Subjekte im Wirtschaftsunterricht geht nicht zwangsläufig gleichzeitig auch eine Ungleichbehandlung durch die Lehrkräfte einher.

\subsubsection{Einbezug sozialer Herkunft}

Im fallübergreifenden Vergleich lässt sich weiterhin der Einbezug der sozialen Herkunft darstellen. Dieser ist innerhalb der Fälle an situationsabhängige Erzählsequenzen gebunden. Vor dem Hintergrund dieser situationsabhängigen Kontexte werden subjektive Theorien konkret, die diesen Einbezug sozialer Herkunft im Wirtschaftsunterricht herausstellen. Exemplarisch für die Kategorie ist die Äußerung von Lehrkraft A:

„Das ist schon eine Problematik für mich die dahinter steckt, auf der anderen Seite ist es schon wichtig wenn die Hälfte der Eltern in der Klasse wenn die arbeitslos ist dann kann ich nicht ständig auf die Arbeitswelt abzielen sondern muss auch andere Dinge mit einbringen. Auf der einen Seite versuche ich immer so das soziale Umfeld gar nicht so genau zu analysieren weil mich das unfrei macht manchmal.“

Hier wird auf die unterrichtliche Situation einer Klasse gegenüberzustehen Bezug genommen, in der die Hälfte der Eltern arbeitslos ist. Als handlungsleitender Mechanismus wird dabei letztlich das Ausblenden der Relevanz sozialer Herkunft beschrieben (vgl. Berg 2014, S. 12). Dabei finden sich innerhalb der Interviews Aussagen zur familiären Sozialisation und insbesondere über die Erwerbstätigkeit der Eltern wieder. Exemplarisch hierzu äußert sich Lehrkraft B wie folgt:

„Sollte es nicht, es ist wichtig zu wissen wo sie herkommen damit man weiß wo man andocken kann, [...] das macht die Sache spannend und es macht möglicherweise auch Verhaltensweisen transparenter von Schülern wenn man weiß, ok der kommt da aus einer Familie wo man weiß wo nur die Mama arbeitet, den Papa gibt es nicht. Manchmal hab ich auch das Gefühl das wir Lehrer das auch sehen wo man herkommt."

Gerade dies ist vor dem Hintergrund der Fragestellung in diesem Beitrag problematisch: Wenn Lehrkraft B beschreibt ,Manchmal hab ich auch das Gefühl dass wir Lehrer das auch sehen wo man herkommt. " lässt sich dies mit Bourdieu als Interpretationsschema auf den Habitus der Schüler_innen und das implizite Wissen über die habitusseitig vermittelte Klassenzügehörigkeit deuten (vgl. Berg 2014, S. 13). Gerade das kann dann als Indiz für die oben beschriebenen objektiven Strukturen gelesen werden, die in der sozialen Welt die Praktiken unabhängig vom Bewusstsein und Willen der Lehrperson leiten. Zudem lässt sich innerhalb des Einbezugs sozialer Herkunft herausstellen, dass der lehrerseitige Rückgriff auf die Begabungsvorstellungen und Vorstellungen über das intellektuelle Potential (vgl. Kap. 2) der Schü- 
ler_innen und die Relevanz für den Wirtschaftsunterricht, erfolgt. Exemplarisch dazu folgende Äußerung von Lehrkraft D:

„Natürlich wenn man sieht untere Schichten sind manche von denen sind nicht so vielleicht ganz begabt oder nicht so auffassungsschnell“.

Diese subjektiven Theorien drücken sich dann auch auf Ebene des Wirtschaftsunterrichts anhand der Frage, wie mit ähnlichen wirtschaftlichen Bildungsinhalten in den verschiedenen Bildungsgängen der berufsbildenden Schule umgegangen wird, aus. Exemplarisch hierzu Lehrkraft A:

„Also Umgang mit Geld ist ja ein Thema und mit der Berufsfachschule gehe ich dann zum Beispiel ins Internet und wir eröffnen ein Konto. Da gibt es bei vielen Banken oder Bankinstituten die Möglichkeit so ein virtuelles Konto zu führen [...] und erstaunlicherweise haben viele noch kein Konto sondern immer von Mama oder Papa. Manchmal leihen sie die Karte aus aber das ist schon erstaunlich was da alles so läuft. Und dann machen wir dass über gemeinsames Ausfüllen, praktische Beispiele lernen sie eben damit umzugehen. In der Berufsfachschule 3 also die [...] bis zur Fachhochschulreife gehen da gehen wir dann dazu schonmal ein Stück weiter dass wir uns dann anschauen: wie laufen dann solche Geschäfte bei der Bank ab, was ist zu beachten wenn man einen Dauerauftrag abgibt, was bedeutet es wenn man ein Konto überzieht, wie wird das berechnet die Zinsen, was sind geduldetet Überziehungskredite und so. Und wenn man, wenn ich das jetzt im Fachgymnasium machen würde, würde ich dann wahrscheinlich über den Umgang mit Geld, Schuldnerberatung, welche Verpflichtung ist da von Seiten des Staates auch mit Menschen umzugehen die mit Geld nicht umgehen können also da so soziale Aspekte mit reinzubringen. Aber alle lernen was ist ein Konto.“

Zwar liegt in der Natur der Sache, dass didaktische Reduktionen je nach Bildungsgang vorgenommen werden, jedoch existieren innerhalb der vorliegenden Schilderung über das Thema Kontoeröffnung bei dieser Lehrkraft anscheinend subjektive Theorien darüber vor, welche Gegenstände welchem Schülerklientel zuzutrauen sind und damit auch wichtig für die Schüler_innen. So dringen die Schüler_innen der Berufsfachschule gar nicht erst zum Beispiel zu den Fragen der Überziehungs-zinsberechung durch. Des Weiteren ordnet diese Lehrkraft soziale Fragen wie Verpflichtungen des Staates oder Schuldnerberatungen den Schüler_innen aus dem Bildungsgang des Gymnasiums zu. Gleichzeitig weden auch subjektive Theorien, die die soziale Herkunft und auch die familiären Sozialisationsbiographien der Schüler_innen in Verbindung mit dem Wirtschaftsunterricht thematisieren, deutlich. Exemplarisch hierfür ist dieses Zitat von Lehrkraft C:

„Wenn Schüler im BFS 1 Bereich erstmal ihre Seiten wechseln müssen eben vom Konsumenten zum Anbieter. Und das ich natürlich bei denen erkenne, die das relativ - oder vermute, dass die diesen Positionswechsel gut hinbekommen, dass sie sozusagen wissen was die Eltern machen oder oder ein Elternteil macht und dass das sozu- 
sagen nichts völlig Fremdes ist. Dafür muss man zumindest im Beruf stehen und das ist bei denen, die eben - aber das ist jetzt vielleicht auch nur ein Vorurteil - die Transferleistungsempfänger sind, ist das mindestens ebenso - also ist der Positionswechsel natürlich genauso wichtig - aber das ich vermute mal das es eher vielleicht dann schwerer fällt $[\ldots]$. "

Hier wird von der Lehrkraft ein Rückgriff auf subjektive Theorie deutlich, indem vermutet wird, dass Schüler_innen deren Eltern Transferleistungsempfänger sind, aufgrund der familiären Herkunft und Sozialisation weniger gut in der Lage sind, den Positionswechsel vom Konsumenten zum Anbieter zu vollziehen.

\section{Diskussion und Schlussbemerkung}

Aus Perspektive der praxeologischen Handlungstheorie werden die Strukturen der Gesellschaft auf der Ebene von sozialen Praktiken angesiedelt. Deutungsmuster, Sinnstrukturen und kollektive Wissensschemata können so erst ihre Wirkung entfalten und überhaupt bestehen (vgl. Moebius 2008, S. 61). Die soziale Praxis ist nicht eine Summe von Einzelhandlungen, sondern ein Teil von übersubjektiven Handlungsgefügen, was dazu führt, dass Sinnstrukturen stabil bleiben und reproduziert werden (ebd.). Denken wir in diesem Zusammenhang nun an die Lebenssituationsorientierung aus fachdidaktischer Perspektive, kommt bei den den befragten Lehrkräften das Anknüpfen an das Subjekt nicht vor Insbesondere, dass die soziale Herkunft zunächst als nicht relevant für den eigenen Unterricht (vgl. Kap. 4.2.1) und offenbar negativ belegt wird, spricht in diesem Kontext für die kognitiv begründbare Handlungslogik das nicht-berücksichtigen der sozialen Herkunft (vgl. Berg 2014, S. 13). Mit Bourdieu lässt sich genau an dieser Stelle eine „Logik der Praxis“ beschreiben, die Interpretationsansätze für eine Reproduktion sozialer Ungleichheiten über die Denk- Wert- und Urteilsmuster der Lehrkräfte bietet. Innerhalb der empirischen Analyse lassen sich Ansatzpunkte dafür identifizieren - einerseits negieren die befragten Lehrkräfte, dass die soziale Herkunft der Schüler_innen relevant ist (vgl. Kap. 4.2.1) und beziehen sich darauf, alle Schüler_innen gleich zu behandeln (vgl. Kap. 4.2.2). Andererseits existieren implizit Vorstellung von Sozialisationshintergründen und Inhalten, die für verschiedene Klientele relevant sind, eben eher ausge-blendet werden sollten oder in unterrichtlicher Konstruktion gut oder weniger gut funktionieren (vgl. Kap. 4.2.3). Mit Bourdieus Konzept des sozialen Raums lässt sich beschreiben, dass diese Lehrkräfte wohl wissentlich auch mit Subjekten konfrontiert sind, die ihnen sozial eher nicht nahe stehen (vgl. Berg 2014, S. 13). Auf einer Handlungsebene entsteht hier dann aber seitens der Lehrkraft Unsi- 
cherheit über den Umgang mit sozialer Herkunft die in willentlicher Ausblendung des Faktors soziale Herkunft mündet. Letztlich greifen aber genau in diesem Moment der Anwendung subjektiver Theorien eben unbewusste habitusbedingte Mechanismen.

\section{Literatur}

Aprea, C. (2011): Alltagsvorstellungen von Jugendlichen zu komplexen ökonomischen Phänomenen: Eine explorative Untersuchung am Beispiel der Wirtschafts- und Finanzkrise. In: Retzmann, T.(Hrsg.): Finanzielle Bildung in der Schule. Mündige Verbraucher durch Konsumentenbildung. Schwalbach/Ts: Wochenschau, S. 100-113.

Bauer, U. (2011): Sozialisation und Ungleichheit. Eine Hinführung. 1. Aufl. Wiesbaden: VS.

Berg, S. (2014): Zur Relevanz von Sozialisationshintergrund und sozialer Herkunft für den Wirtschaftsunterricht. Vorstellung von Handelslehrern über die Perspektive der Lernenden. In: bwp@ Berufs- und Wirtschaftspädagogik - online, Ausgabe 26, 1-17. Online: http://www.bwpat.de/ausgabe26/berg_bwpat26.pdf (20-06-2014).

Birke, F./Seeber, G. (2011): Heterogene Schülerkonzepte für ökonomische Phänomene - ihre Erfassung und Konsequenzen für den Unterricht. In: Journal of Social Science Education, 10. Bd., H. 2, S. 56-66.

Bourdieu, P. (1974): Zur Soziologie der symbolischen Formen. Frankfurt a. M.: Suhrkamp.

Bourdieu, P. (1992): Rede und Antwort. Frankfurt a. M.: Suhrkamp.

Bourdieu, P. (1995): Sozialer Raum und Klassen. Frankfurt a. M.: Suhrkamp.

Bourdieu, P. (2012): Die feinen Unterschiede. Kritik der gesellschaftlichen Urteilskraft. 22. Aufl. Frankfurt a. M.: Suhrkamp.

Ditton, H. (2007): Der Beitrag von Schule und Lehrern zur Reproduktion von Bildungsungleichheit. In: Becker, R. (Hrsg.): Bildung als Privileg. Erklärungen und Befunde zu den Ursachen der Bildungsungleichheit. 2., aktualisierte Aufl. Wiesbaden: VS, S. 243-271.

Ditton, H. (2009): Familie und Schule - eine Bestandsaufnahme der bildungssoziologischen Schuleffektforschung von James S. Coleman bis heute. In: Becker, R. (Hrsg.): Lehrbuch der Bildungssoziologie. 1. Aufl. Wiesbaden: VS, S. 239-259.

Dubs, R. (2001): Widerspruch oder produktives Spannungsverhältnis zwischen Praxis- und Wissenschaftsbezug? In: bwp@ Berufs- und Wirtschaftspädagogik, 1. Bd.

Euler, D./Hahn, A. (2004): Wirtschaftsdidaktik. Bern: Haupt.

Flick, U. (2008): Triangulation: Eine Einführung. 2. Aufl. Wiesbaden: VS.

Flick, U./Kardorff, E./Steinke, I. (Hrsg.) (2012): Qualitative Forschung. Ein Handbuch. Orig.-Ausg., 9. Aufl. Reinbek bei Hamburg: Rowohlt-Verlag.

Geißler, R. (2012): Die meritokratische Illusion - oder warum Reformen beim Bildungssystem ansetzen müssen. In: Haller, M./Niggeschmidt, M. (Hrsg.): Der 
Mythos vom Niedergang der Intelligenz. Von Galton zu Sarrazin: Denkmuster und Denkfehler der Eugenik.: VS, S. 193-210.

Groeben, N./Wahl, D./Schlee, J./Scheele, B. (1988): Das Forschungsprogramm Subjektive Theorien. Eine Einführung in die Psychologie des reflexiven Subjekts. Tübingen: Francke.

Joas, H./Knöbl, W. (2011): Sozialtheorie. Zwanzig einführende Vorlesungen. Aufl. Frankfurt a. M.: Suhrkamp.

Kramer, R.-T. (2011): Abschied von Bourdieu? Perspektiven ungleichheitsbezogener Bildungsforschung. Wiesbaden: VS.

Kramer, R. T./Helsper, W. (2011): Kulturelle Passung und Bildungsungleichheit Potenziale einer an Bourdieu orientierten Analyse der Bildungsungleichheit. In: Krüger, H.-H./Rabe-Kleberg, U./Kramer, R.-T./Budde, J (Hrsg.): Bildungsungleichheit revisited. Bildung und soziale Ungleichheit vom Kindergarten bis zur Hochschule. Wiesbaden: VS, S. 103-126.

Kühne, S. (2006): Das soziale Rekrutierungsfeld der Lehrer. Empirische Befunde zur schichtspezifischen Selektivität in akademischen Berufspositionen. In: Zeitschrift für Erziehungswissenschaften 9. Bd., H. 4, S. 617-631.

Moebius. S. (2008): Handlung und Praxis: Konturen einer poststrukturalistischen Praxistheorie. In: Moebius, S./Reckwitz, A. (Hrsg.): Poststrukturalistische Sozialwissenschaften. 1. Aufl. Frankfurt a. M.: Suhrkamp.

Retzmann, T./Seeber G./Remmele B./Jongebloed, H.-C. (2010): Ökonomische Bildung an allgemeinbildenden Schulen. Bildungsstandards. Standards für die Lehrerbildung. Essen, Lahr, Kiel.

Roland-Lévy, C. (2002): Economic Socialisation. How Does One Develop an Understanding of the Economic World? In: Hutchings, M./Fülöp, M./van den dries, A.M. (Eds.) Young People's Understanding of Economic Issues in Europe: Stoke on Trent. S. 17-30.

Seifried, J. (2009): Unterricht aus der Sicht von Handelslehrern. Frankfurt a. M., Bern: P. Lang.

Seifried, J./Sembill, D. (2005): Emotionale Befindlichkeit in Lehr-Lern-Prozessen in der beruflichen Bildung. In: Zeitschrift für Pädagogik 51. Bd., H. 5, S. 656-672.

Schroer, M. (2008): Raum oder: Das Ordnen der Dinge. In: Moebius, S./Reckwitz, A. (Hrsg.): Poststrukturalistische Sozialwissenschaften. Frankfurt a. M.: Suhrkamp, S. 141-157.

Seeber, G./Remmele, B. (2009): Ökonomische Kompetenz bedingt Einstellungen zur Marktwirtschaft - Entwurf eines Wirkmodells zur empirischen Analyse. In: Seeber, G. (Hrsg.): Forschungsfelder der Wirtschaftsdidaktik. Herausforderungen - Gegenstandsbereiche - Methoden. Frankfurt a. M.: Wochenschau-Verlag, S. 27-40.

Strauss, A. L. (1991): Grundlagen qualitativer Sozialforschung - Datenanalyse und Theoriebildung in der empirischen soziologischen Forschung. München: Fink. 